\title{
Nonlinear differential algorithm to compute all the zeros of a generic polynomial
}

\author{
Francesco Calogero \\ Physics Department, University of Rome "La Sapienza", 00185 Rome, Italy \\ Istituto Nazionale di Fisica Nucleare, Sezione di Roma
}

Abstract

A simple algorithm to compute all the zeros of a generic polynomial is proposed.

\section{Introduction}

Notation. Hereafter, for definiteness, we always refer to monic polynomials of arbitrary order $N(N \geq 2)$,

$$
P_{N}(z ; \vec{c}, \underline{x})=z^{N}+\sum_{m=1}^{N}\left(c_{m} z^{N-m}\right)=\prod_{n=1}^{N}\left(z-x_{n}\right) ;
$$

the complex variable $z$ is the argument of the polynomial, indices such as $n, m, \ell$ run from 1 to $N$ (unless otherwise indicated, see below), the $N$-vector $\vec{c}$ has the $N$ coefficients $c_{m}$ of the polynomial (10) as its $N$ components, $\underline{x}$ is the unordered set of the $N$ zeros $x_{n}$ of the polynomial (1), and we assume all variables to be complex (unless otherwise explicitly indicated, see below). We call generic any polynomial the coefficients and zeros of which are generic complex numbers, and in particular feature zeros which are all different among themselves, $x_{n} \neq x_{m}$ if $n \neq m$. Note that the notation $P_{N}(z ; \vec{c}, \underline{x})$ is somewhat redundant, since this monic polynomial can be identified by assigning either its $N$ coefficients or its $N$ zeros; indeed the $N$ coefficients $c_{m}$ can be expressed in terms of the $N$ zeros $x_{n}$ via the following standard formula

$$
c_{m}=(-1)^{m} \sum_{n_{1}>n_{2}>\ldots>n_{m}=1}^{N}\left(x_{n_{1}} x_{n_{2}} \cdots x_{n_{m}}\right),
$$

so that

$$
\begin{gathered}
c_{1}=-\left(x_{1}+x_{2}+\ldots+x_{N}\right), \\
c_{2}=\left(x_{1} x_{2}+x_{1} x_{3}+\ldots+x_{1} x_{N}\right) \\
+\left(x_{2} x_{3}+x_{2} x_{4}+\ldots+x_{2} x_{N}\right)+\ldots \\
+\left(x_{N-2} x_{N-1}+x_{N-2} x_{N}\right)+x_{N-1} x_{N},
\end{gathered}
$$

and so on. On the other hand, while the assignment of the $N$ coefficients $c_{m}$ determines the $N$ zeros $x_{n}$-uniquely, up to permutations - of course explicit formulas to accomplish generally this task only exist for $N \leq 4$. 
The investigation of the properties - and of techniques for the numerical computation - of the $N$ zeros $x_{n}$ of a polynomial of degree $N$ defined via the assignment of its $N$ coefficients $c_{m}$ (see (10) is a problem that has engaged mathematicians since time immemorial. In this paper a simple nonlinear differential algorithm suitable to compute numerically all the $N$ zeros of a generic polynomial of arbitrary degree $N$ is described; I was unable to find a previous description of this algorithm in the literature, but I am aware that my search has not been - indeed, it could not have been - quite complete. This algorithm is described in the following Section 2 and proven in Section 3.

\section{Results}

It is now convenient to introduce an additional independent variable $t$, which is hereafter assumed to be real and might be interpreted as time. Hence the above notation is now extended by writing, in addition to (1), the analogous formula

$$
p_{N}(z ; \vec{\gamma}(t), \underline{y}(t))=z^{N}+\sum_{m=1}^{N}\left[\gamma_{m}(t) z^{N-m}\right]=\prod_{n=1}^{N}\left[z-y_{n}(t)\right],
$$

to which notational comments quite analogous to those reported above apply.

There holds then the following

Proposition. Consider the following system of $N$ nonlinear first-order differential equations satisfied by the $N$ zeros $y_{n}(t)$ of the polynomial (3):

$$
\dot{y}_{n}(t)=-\left\{\prod_{\ell=1, \ell \neq n}^{N}\left[y_{n}(t)-y_{\ell}(t)\right]^{-1}\right\} \sum_{m=1}^{N}\left\{\left[c_{m}-\gamma_{m}(0)\right]\left[y_{n}(t)\right]^{N-m}\right\} .
$$

Here and below a superimposed dot denote a $t$-differentiation, while the coefficients $c_{m}$ are those of the polynomial $P_{N}(z ; \vec{c}, \underline{x})$, see (1), the zeros $x_{n}$ of which we seek, and $\gamma_{m}(0)$ are the $N$ coefficients of the polynomial $p_{N}(z ; \vec{\gamma}(t), \underline{y}(t))$, see (3), at $t=0$, hence they are related to the "initial" values $y_{n}(0)$ of the zeros of this polynomial by the formula (analogous to (2))

$$
\gamma_{m}(0)=(-1)^{m} \sum_{n_{1}>n_{2}>\ldots>n_{m}=1}^{N}\left[y_{n_{1}}(0) y_{n_{2}}(0) \cdots y_{n_{m}}(0)\right] .
$$

Then

$$
x_{n}=y_{n}(1) .
$$

It is thus seen that the zeros $x_{n}$ of the polynomial $P_{N}(z ; \vec{c}, \underline{x})$, see (11), can be computed - once the $N$ coefficients $c_{m}$ of this polynomial have been assignedvia the following procedure. Step one: choose (arbitrarily!) $N$ complex numbers $y_{n}(0)$. Step two: compute, via the formulas (4b), the $N$ quantities $\gamma_{m}(0)$. Step

three: integrate (numerically) the system of differential equations (4a) from 
$t=0$ to $t=1$, starting from the $N$ initial data $y_{n}(0)$, getting thereby the $N$ values $y_{n}(1)$, which give the sought result, see (4c).

Will this procedure always work? The only possible snag is that the solution $\vec{y}(t)$ of the "dynamical system" (4a) run into a singularity during its evolution from $t=0$ to $t=1$. The only mechanism whereby this might occur is because during this evolution two different coordinates $y_{n}(t)$ might coincide, $y_{\ell}(t)=$ $y_{n}(t)$ for $\ell \neq n$, at some value of the real variable $t$ in the interval $0<t<1$, causing the right-hand side of (4a) to blow up. This "collision" might indeed happen, but it is not a generic phenomenon: hence it will be enough to change the assignment of the (arbitrary!) initial data $y_{n}(0)$ to avoid this difficulty; note however that this suggests that to apply this method it will be advisable to always start with complex initial data $y_{n}(0)$, even in the case of real polynomials with real zeros. And note that by performing the numerical integration of the differential equations (4a) with different initial data $\vec{y}(0)$ provides moreover a possibility to assess the numerical accuracy of the computation, by comparing the results obtained starting from different sets of initial data.

Remark. It is plain that this procedure will work more efficiently the closer the, arbitrarily chosen, initial values $y_{n}(0)$ are to the $N$ zeros $x_{n}$ the values of which one is trying to compute; indeed if the $N$ initial values $y_{n}(0)$ happened to coincide with the $N$ zeros $x_{n}, y_{n}(0)=x_{n}$, this would imply $\gamma_{m}(0)=c_{m}$ (compare (2) with (4b) ) hence the right-hand side of the differential equations (4a) would vanish identically, entailing $\dot{y}_{n}=0$ hence $y_{n}(1)=y_{n}(0)=x_{n}$, consistently with (4c).

Let us also emphasize that the dependence (via (4b)) of the right-hand sides of the differential equations (4a) upon the initial values $y_{n}(0)$ of the dependent variables $y_{n}(t)$ implies that these differential equations are rather Differential Functional Equations than Ordinary Differential Equations; but this fact has hardly any relevance on step three of the procedure, see above.

A comparison of the actual effectiveness of this technique with that of other methods to compute all the $N$ zeros of a generic polynomial of arbitrary degree $N$ is beyond the scope of this short communication, and in any case it is a task to be rather pursued by specialists in numerical analysis if they consider it worthy of their attention.

\section{Proof}

The proof of the above Proposition is actually quite easy (raising thereby some doubts on the novelty of this finding). The starting point is the identity

$$
\dot{y}_{n}(t)=-\left\{\prod_{\ell=1, \ell \neq n}^{N}\left[y_{n}(t)-y_{\ell}(t)\right]^{-1}\right\} \sum_{m=1}^{N}\left\{\dot{\gamma}_{m}(t)\left[y_{n}(t)\right]^{N-m}\right\},
$$

valid for any $t$-dependent polynomial with zeros $y_{n}(t)$ and coefficients $\gamma_{m}(t)$, 
see (3); for a proof of this formula see [1]. Now make the assignment

$$
\gamma_{m}(t)=\gamma_{m}(0)+\left[c_{m}-\gamma_{m}(0)\right] t,
$$

consistent with the initial (arbitrary) assignment at $t=0$ and clearly implying

$$
\begin{gathered}
\dot{\gamma}_{m}(t)=c_{m}-\gamma_{m}(0), \\
\gamma_{m}(1)=c_{m} .
\end{gathered}
$$

The insertion of the first of these two formulas, (6b), in (5) yields (4a); while the second, (6c), implies that, at $t=1$, the polynomial $p_{N}(z ; \vec{\gamma}(t), \underline{y}(t))$, see (3), coincides with the polynomial $P_{N}(z ; \vec{c}, \underline{x})$, see (11), hence the validity of (4c). Q. E. D.

\section{References}

[1] F. Calogero, "New solvable variants of the goldfish many-body problem", Studies Appl. Math. (in press, published online 07.10.2015). DOI: 10.1111/sapm.12096. 